\title{
Undulator configuration for helicity switching in in-vacuum undulators
}

\author{
Ryota Kinjo॰* and Takashi Tanaka \\ RIKEN SPring-8 Center, 1-1-1, Koto, Sayo-cho, Sayo-gun, Hyogo, 679-5148, Japan
}

(Received 12 December 2019; accepted 12 February 2020; published 21 February 2020)

\begin{abstract}
We propose a new scheme to control the polarization state of undulator radiation, in which the helicity of circularly polarized radiation can be switched by translating the whole undulator along the horizontal direction. This is in contrast to the conventional method based on a phasing operation (longitudinal motion of magnetic arrays), which gives rise to a large variation of the three-dimensional magnetic force acting on the magnetic array. Because the mechanical design can be much simpler than conventional undulators equipped with a function for the phasing operation, the proposed scheme is well compatible with invacuum undulators. Numerical and experimental studies were carried out to reveal the performance and feasibility of the proposed scheme, showing its potential as an attractive option for the in-vacuum undulators.
\end{abstract}

DOI: 10.1103/PhysRevAccelBeams.23.020705

\section{INTRODUCTION}

Polarization control is one of the key technologies in synchrotron radiation and $\mathrm{x}$-ray free electron laser applications, and various schemes have been proposed and developed to date [1-9]. Among them, the so-called APPLE undulator [3] has been widely chosen because of its flexibility in polarization control, in which any polarization state (horizontal, vertical, inclined linear, right handed, or left handed) is in principle available.

The polarization control of the APPLE undulator is based on a mechanical operation; the top and bottom magnetic arrays are horizontally split into two arrays that are mounted on two different girders, and a diagonal pair of girders (and thus magnetic arrays) is moved along the longitudinal direction to tune the polarization state of radiation, which is usually referred to as "phasing".

The most critical issue in designing the APPLE undulator is how to deal with the large magnetic force acting on the girders and magnetic arrays, especially during the phasing operation. In contrast to the magnetic-force variation during the gap operation in a conventional planar undulator, which is one dimensional (vertical) and monotonic (exponential), the force variation during the phasing operation is three dimensional and not monotonic. This imposes a stringent requirement on the mechanical

\footnotetext{
r-kinjo@spring8.or.jp
}

Published by the American Physical Society under the terms of the Creative Commons Attribution 4.0 International license. Further distribution of this work must maintain attribution to the author(s) and the published article's title, journal citation, and DOI. specifications such as the stiffness of the girder and supporting frame, and the torque of the driving motor.

The aforementioned issues coming from the phasing operation have made it critically challenging to develop an "APPLE in-vacuum undulator (IVU)". In spite of its potential to extend the wavelength tunability and impact on the applications of polarized synchrotron radiation and $\mathrm{x}$-ray free electron laser, APPLE IVUs have never been put into practical use at least to our knowledge.

Recently, Bahrdt and Grimmer have proposed a design [10] to overcome the difficulty toward the realization of APPLE IVUs, which is based on magnetic force compensation with additional magnetic arrays, and facilitates the phasing operation in IVUs. Although this concept will offer a new scheme to implement the polarization control in IVUs, the whole structure will be much more complicated than conventional IVUs and its realization seems to be still challenging. In this paper, we present an alternative approach toward the realization of polarization control in IVUs. Although the available polarization state is limited compared to the conventional APPLE undulator, the structure and operation of the undulator based on the proposed scheme are much simpler, and thus it can be easily implemented in IVUs. In addition, the magnetic force in the proposed undulator can be reduced to a negligible level by carefully optimizing the dimensions and positions of magnet arrays, without any special equipment.

\section{PRINCIPLE}

Let us first recall how the polarization control works in the APPLE undulators, together with the difficulty to be solved for application to IVUs. In what follows, $z$ denotes the longitudinal direction along which the electron beam is injected, while $x$ and $y$ denote the horizontal and vertical 


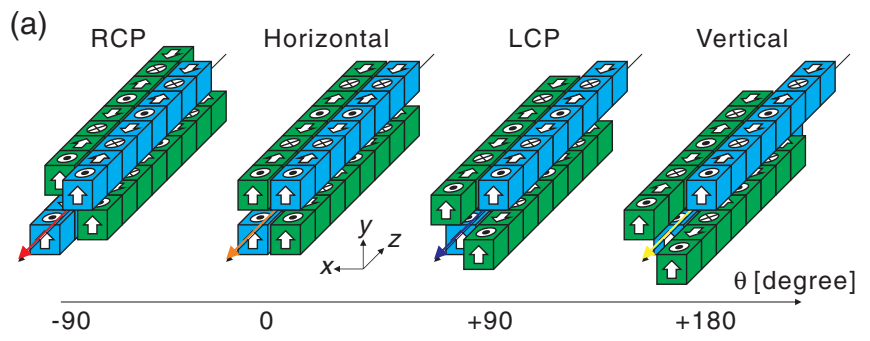

(b)

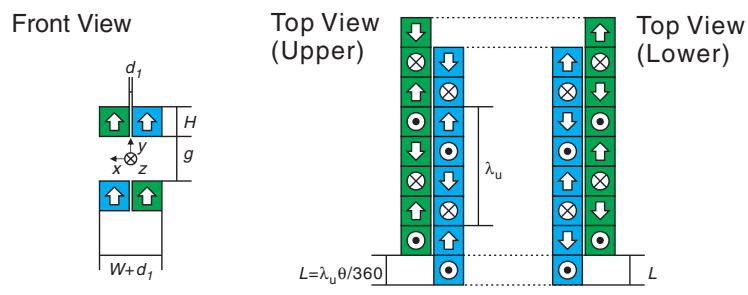

FIG. 1. Outline of the APPLE undulator: (a) magnet configurations to generate four typical polarization states, and (b) geometry to calculate the magnetic force.

directions perpendicular to $z$. Figure 1(a) illustrates the magnet configurations of the APPLE undulator, in which the diagonal pair of magnetic arrays is painted with the same color (green and blue), and the phase $\theta$ is defined as

$$
\theta=\frac{L}{\lambda_{u}} \times 360^{\circ}
$$

with $\lambda_{u}$ being the undulator period, and $L$ being the relative distance between the diagonal pairs along $z$.

In this configuration, the diagonal pair is moved in the same direction to control the polarization state; for example, horizontal, vertical, right-handed, and left-handed polarizations are available with $\theta=0,180^{\circ},-90^{\circ}$ and $90^{\circ}$, respectively. For convenience, the configurations for $\theta=$ $\pm 90^{\circ}$ are referred to as the RCP (right-handed circular polarization) and LCP (left-handed circular polarization) configurations, respectively, although the resultant polarization may not be exactly circular. Note that the so-called antiparallel mode, in which the diagonal pair is moved in the opposite direction to generate linearly polarized radiation with an arbitrary tilt angle, is not considered in this paper.

As mentioned in the Introduction, the phasing operation gives rise to a large variation of the magnetic force. For example, Figs. 2(a) and 2(b) plot the magnetic force $\left(F_{x}, F_{y}\right.$, and $\left.F_{z}\right)$ as a function of the phase $\theta$, for an APPLE undulator with the remanent field of $1.2 \mathrm{~T}$, and $H=\lambda_{u}=18 \mathrm{~mm}, W=15 \mathrm{~mm}, d_{1}=1 \mathrm{~mm}$, where the definitions of these parameters are given in Fig. 1(b). Note that the magnetic force is defined as that acting on one of the four arrays, which is located at the top-right side, and was numerically calculated using the code RADIA [11].

When the gap is narrow $(2 \mathrm{~mm})$, the magnetic force significantly varies as the phase, with the maximum value reaching several $\mathrm{kN} / \mathrm{m}$ in all the directions. Although the vertical component $\left(F_{y}\right)$ vanishes when the gap is wide $(20 \mathrm{~mm})$ as in the conventional planar undulator, the other two components keep a similar level. This is attributable to the narrow horizontal gap between the two magnetic arrays, and imposes a stringent requirement on the mechanical specifications of the APPLE undulator.

The three-dimensional magnetic force and its variation during the phasing operation as described above, also become the biggest obstacle to the realization of APPLE IVUs. In addition, the phasing operation poses a critical issue regarding the vacuum components to reduce the impedance; they are specific to IVUs in which the electron beam is surrounded by a huge number of magnet blocks held by the in-vacuum girders located inside the vacuum chamber. It is obvious that the conventional IVU technologies [12] such as the Ni-coated copper sheet to cover the magnet surface, and a flexible taper system to smoothly

(b)

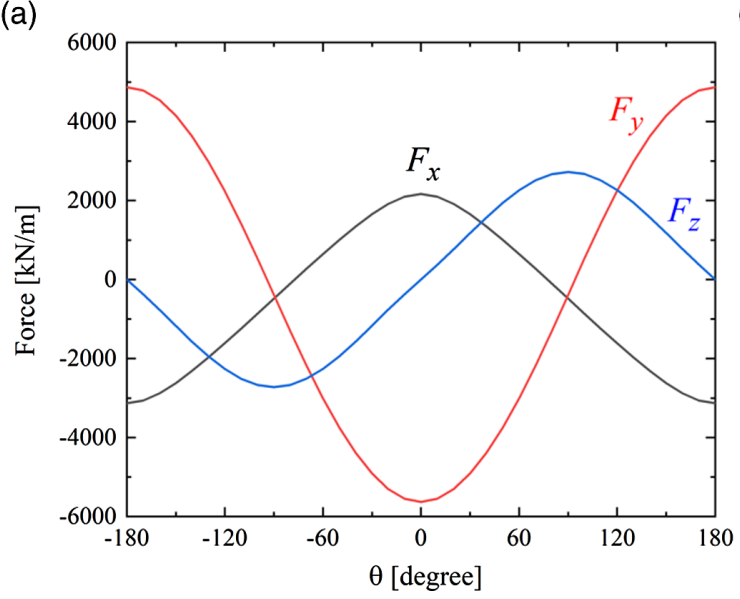

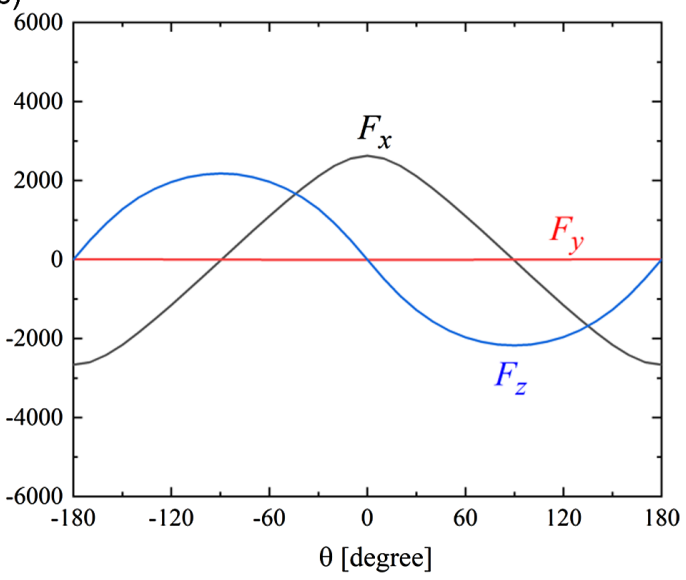

FIG. 2. Magnetic force of an APPLE undulator calculated as a function of the phase for two different gap values of (a) 2 mm and (b) $20 \mathrm{~mm}$. 
connect the end of the magnetic array to the aperture of the vacuum duct, are not available any more; we need to establish an alternative technology compatible with the phasing operation. It should be emphasized here that the difficulties mentioned above come from the phasing operation and have nothing to do with the magnetic circuit; in practice, a number of fixed-phase IVUs have been constructed and operated in SPring-8 to generate radiation with a nonhorizontal polarization state (vertical [13], circular with fixed helicity [14]).

Now let us revisit the practical goal of the polarization control. Although the capability to fully manipulate the polarization state, or more specifically to generate radiation with an arbitrary state of polarization, may be advantageous to users, most of the applications do not actually take advantage of it; what they really need is to "switch" the polarization state from one to another. For example, reversing the helicity of circularly polarization radiation is sufficient for the $\mathrm{x}$-ray magnetic circular dichroism experiments. If we discard the possibility to fully manipulate the polarization state and limit the function to the helicity switching, a new undulator configuration alternative to the APPLE one may be possible, which is well compatible with the requirement for IVUs. This is the motivation to propose a new undulator configuration, which is explained as follows.

Let us first recall the RCP and LCP configurations of the APPLE undulator to generate right-handed $\left(\theta=-90^{\circ}\right)$ and left-handed $\left(\theta=90^{\circ}\right)$ polarizations, which are shown again in Figs. 3(a) and 3(b), respectively. Note that several magnet blocks are moved from the downstream/upstream to the upstream/downstream ends to be consistent with the

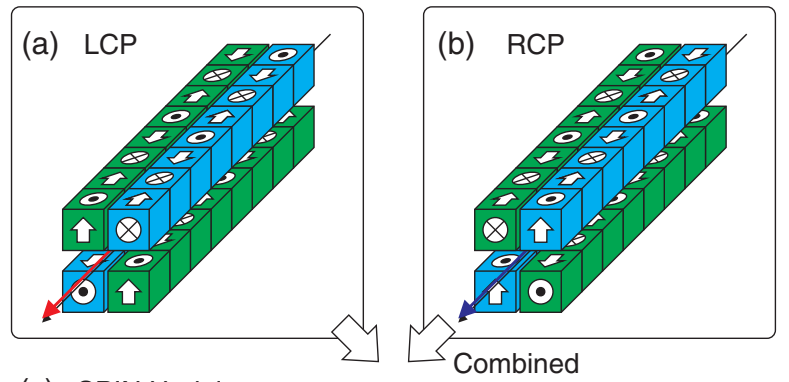

(c) SPIN Undulator

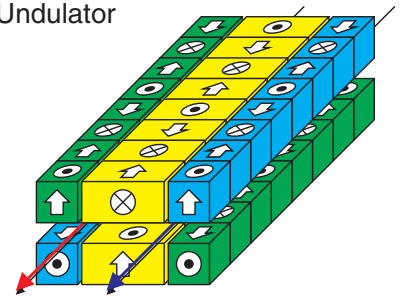

FIG. 3. Schematic illustration of the SPIN configuration: (a) RCP and (b) LCP configurations of the APPLE undulator are combined to form the configuration shown in (c). The helicity can be switched by changing the relative horizontal position between the electron beam and the undulator. following discussion; we emphasize that the periodicity of magnetization vectors and the phase relation between magnetic arrays are still identical to those in Fig. 1(a). It is easy to find that the two arrays in the right side of the RCP configuration shown in Fig. 3(a) are the same as those in the left side of the LCP configuration shown in Fig. 3(b).

Next, we consider a magnet configuration shown in Fig. 3(c), which is given by combining the RCP (a) and LCP (b) configurations. What is important is that all the magnets in the top or bottom array are mounted on a common girder, and thus the phase is fixed. From the discussions above, it is reasonable to expect that the electron beam passing through the axis of the RCP part emits RCP radiation (red arrow), while that of the LCP part emits LCP radiation (blue arrow). Then, the LCP and RCP can be switched by changing the relative horizontal position of the electron beam with respect to the undulator. In other words, two different polarization states are available with a fixed-phase configuration, which is hereafter referred to as "selectable polarization with invariant phase (SPIN)" configuration. It is obvious that applying this concept to IVUs is much easier than the realization of APPLE IVUs. Note that an IVU composed of 6 magnetic arrays has been constructed [15] and operated without any problem, suggesting the feasibility of the magnetic configuration shown in Fig. 3(c).

In addition to the excellent compatibility with IVUs, the SPIN configuration has another important advantage; the magnetic force acting on each magnet array can be eliminated by optimizing the dimensions of magnet blocks and adjustment of the longitudinal position of individual magnetic arrays, which is discussed later in detail. This helps to simplify the mechanical structure of the undulator as presented in [16].

We now discuss practical issues on the performance and operation of IVUs based on the SPIN configuration. In terms of the magnetic performance, the two axes of the RCP and LCP parts should be distant enough to reduce any adverse effect with each other. On the other hand, they should be as close as possible in terms of operation, because the electron beam should go back and forth between the two axes to switch the helicity of radiation. This means that we need to make a compromise, and it is reasonable to say that the typical distance between the two axes to satisfy the above conditions is, roughly speaking, of the order of $\lambda_{u}$, which is usually around $20 \mathrm{~mm}$ or shorter for IVUs. Thus the distance between the two axes will be of the order of a few tens of millimeters.

There are in principle two methods to change the relative position of the electron beam to select the polarization state. One is to generate a bump orbit and the other is to translate the whole undulator in the horizontal direction. From a practical point of view, the former method is unrealistic, because generating a bump orbit with an offset larger than $10 \mathrm{~mm}$ needs a strong dipole field and a long $(\gg 10 \mathrm{~m}) \mathrm{drift}$ 
section for a high-energy $(\mathrm{GeV})$ electron beam, which is usually unacceptable; moreover, the two different optical axes of the RCP and LCP radiations, which are more than $10 \mathrm{~mm}$ away from each other, make any applications practically impossible.

The above discussions suggest that the whole undulator should be translated horizontally to switch the helicity, as schematically illustrated in Fig. 4, where the undulator mechanical frame is mounted on a linear guide to allow for the horizontal motion. Such a system to translate the whole undulator has been actually built and operated [17], although it was an out-vacuum type. It should be noted that the vacuum chamber should be connected to the adjacent vacuum duct with a pair of bellows that allows for the horizontal translation. The switching rate of this scheme is thought to be around the same level as that in the APPLE undulator because the mechanical loads in the SPIN and APPLE undulators are the same level of several tons.

Here, we briefly discuss the measures against the beaminduced wall current. It is well known that two components are usually implemented in IVUs for this purpose: copper foils to cover the surface of the magnetic arrays, and flexible transitions to connect the ends of the magnetic

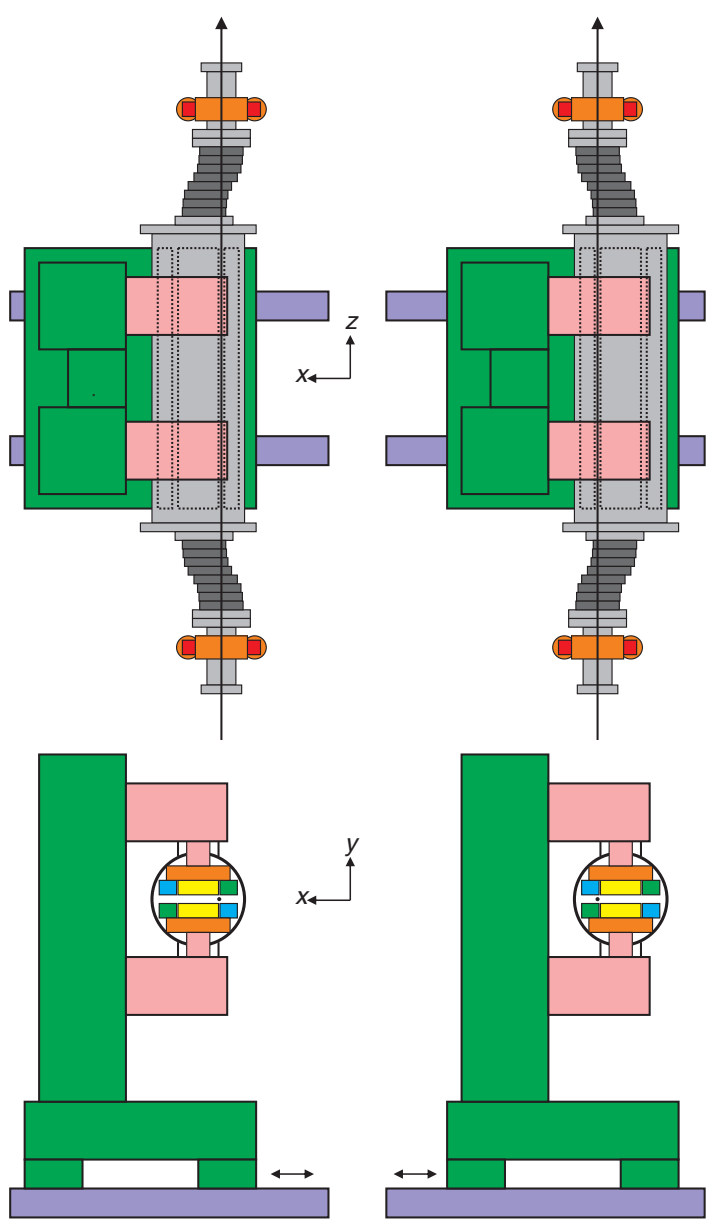

FIG. 4. Switching the helicity by translating the whole undulator. array and vacuum duct. Both of them can be easily modified to be compatible with the SPIN undulators, as long as the end bellows are long enough to allow for the horizontal motion.

\section{NUMERICAL DEMONSTRATION}

Having described the principle, let us numerically evaluate the performance of the proposed SPIN undulator, whose geometry is shown in Fig. 5 together with the parameters to be determined. Note that the top-central, bottom-left, and bottom-right arrays are longitudinally shifted by the distance $\Delta L$ in the same direction, from the original position shown in Fig. 3(c). We also define the additional phase $\Delta \theta$ as

$$
\Delta \theta=\frac{\Delta L}{\lambda_{u}} \times 360^{\circ},
$$

which is also an important parameter to be optimized.

Among the parameters introduced above, we assume $H=\lambda_{u}=18 \mathrm{~mm}$ and $d_{1}=1 \mathrm{~mm}$ to be compared with the APPLE undulator discussed before, and $W=60 \mathrm{~mm}$ as a typical value in IVUs where the magnetic array should fit into a vacuum chamber. The other two parameters, $W_{c}$ and $\Delta \theta$, are optimized to minimize the magnetic force based on numerical calculations performed with RADIA. In the following discussion, the magnetic force in the SPIN configuration is defined as that acting on the top girder holding the three magnetic arrays.

As an example, we show the results of optimization at the gap of $2 \mathrm{~mm}$. Figures 6(a) and 6(b) show the magnetic force calculated as a function of $W_{c}$ and $\Delta \theta$, respectively, with $\Delta \theta=0$ and $W_{c}=30 \mathrm{~mm}$ being fixed. We find that $F_{y}$ hardly depends on $W_{c}$ and $F_{z}$ hardly depends on $\Delta \theta$, meaning that their optimum values are independently determined, namely, $W_{c}=28 \mathrm{~mm}$ and $\Delta \theta=4^{\circ}$. It is worth noting that these optimum values are also effective in terms of the magnetic performance. For example, Fig. 6(c) shows the horizontal and vertical $\mathrm{K}$ values calculated as a function of $\Delta \theta$ with $W_{c}=30 \mathrm{~mm}$, indicating that the condition

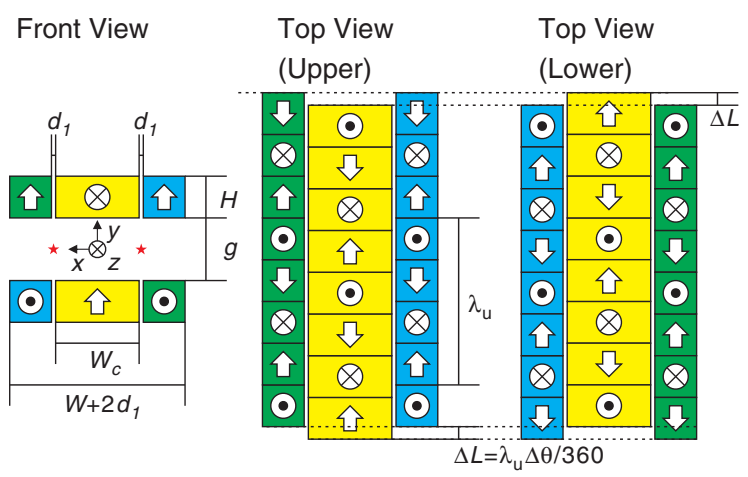

FIG. 5. Geometry to calculate the performance of the SPIN undulator. 


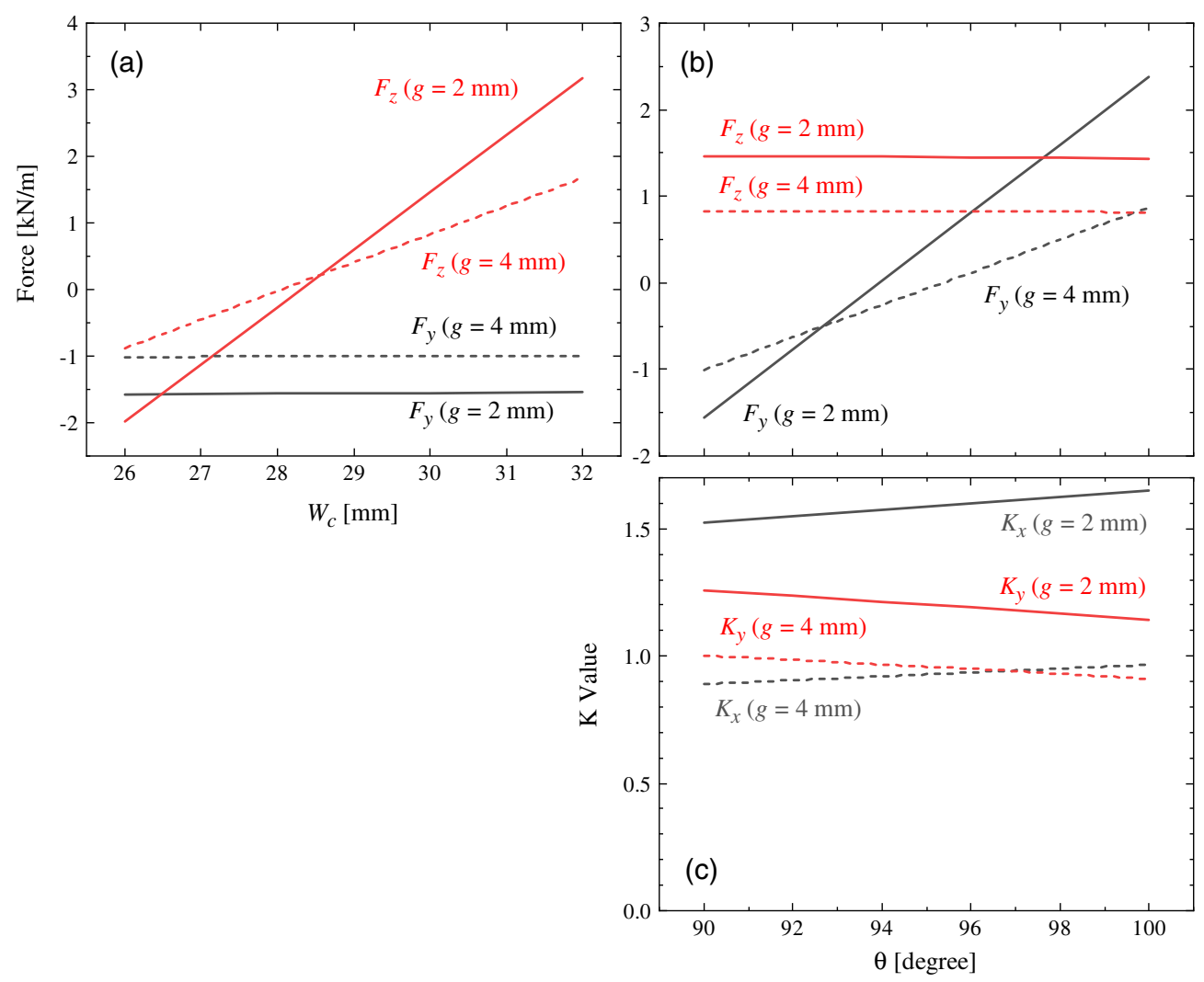

FIG. 6. Results of numerical calculation carried out to demonstrate the performance of the SPIN undulator: (a) and (b) magnetic force vs $W_{c}$ and $\Delta \theta$, and (c) $\mathrm{K}$ values vs $\Delta \theta$.

$K_{x}=K_{y}$, which results in a higher degree of polarization, is roughly satisfied at $\Delta \theta=4^{\circ}$ at least between $g=2 \mathrm{~mm}$ and $g=4 \mathrm{~mm}$.

Using the parameters optimized above, let us make a comparison between the APPLE and SPIN undulators. Figure 7(a) shows the $\mathrm{K}$ values and the degree of circular polarization $P_{c}$ as a function of the gap. Note that $P_{c}$ has been evaluated for on-axis radiation emitted by a single electron, in which the relation between $K_{x}$ and $K_{y}$ is the only parameter to be considered. Note that the phase of the APPLE undulator is optimized at each gap to keep the condition $K_{x}=K_{y}$. This is obviously an advantage of the APPLE undulator against the SPIN undulator in which the above condition is satisfied only at a specific fixed gap and is not kept for other gap values as shown in Fig. 7(a). On the other hand, the SPIN undulator has a great advantage in terms of the mechanical load as shown in Fig. 7(b), where the magnetic force is plotted as a function of the gap. Note that two cases of the magnetic force in the APPLE undulator are plotted; one is the maximum value reached during the phasing operation at each gap (solid) and the other is the force at the optimized phase (empty). The magnetic force of the SPIN undulator is an order of magnitude lower than that of the APPLE undulator and is comparable to the weight of the magnetic arrays and girders. This gives a possibility to significantly reduce the weight of the mechanical frame because of the much lower load than conventional undulators, which is important in designing the mechanical system to translate the whole undulator and switch the helicity. Because not only the force but also the torque is a concern in the mechanical design of the undulator, we also calculated the torque supposed in the operation of the SPIN and APPLE undulators, and found that the former is much lower than the latter.

The effect on the electron beam dynamics is compared between the APPLE and SPIN undulators in terms of horizontal kick map in Fig. 8. Note that the calculation was made with the parameters of $g=4 \mathrm{~mm}$ and $\theta=90^{\circ}$ in both undulators, and the origin in the SPIN undulator is defined as the electron beam axis in the RCP configuration. The effects in the both undulators are found to be similar, and thus we may need to apply some correction schemes. Note that the small residual kicks at the origin, which is found in the SPIN undulator (inset), come from the asymmetry in the magnetic configuration with respect to the electron beam axis.

\section{EXPERIMENTAL DEMONSTRATION}

To experimentally demonstrate the performance of the proposed SPIN configuration, we built a pair of prototype magnetic arrays with the parameters of $\lambda_{u}=H=18 \mathrm{~mm}$, 


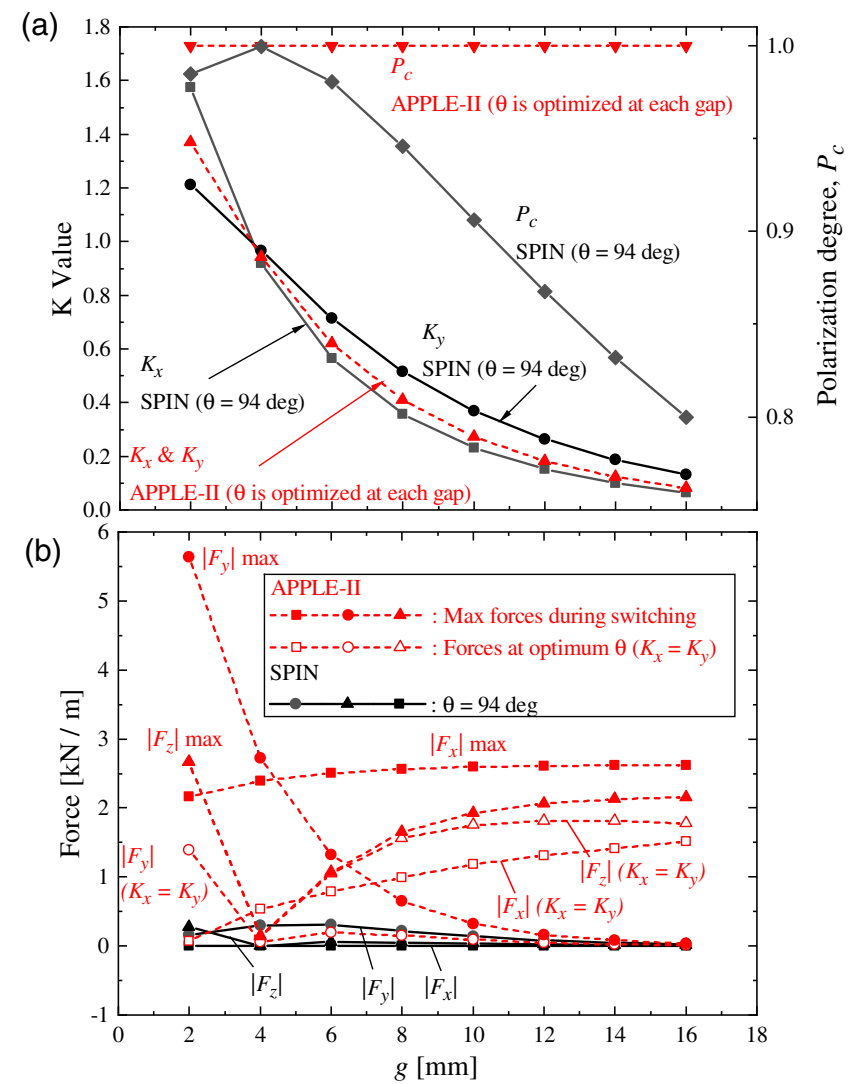

FIG. 7. Comparison between the APPLE and SPIN undulators in terms of (a) the $\mathrm{K}$ values and the degree of circular polarization $P_{c}$, and (b) the magnetic force, calculated as a function of the gap value.

$d_{1}=1 \mathrm{~mm}, W_{c}=30 \mathrm{~mm}$, and $W=60 \mathrm{~mm}$, which corresponds to the top and bottom magnetic arrays of a SPIN undulator with the number of periods of 8 . Note that the longitudinal position of the central array can be manually adjusted, so that the additional phase $\Delta \theta$ is experimentally optimized.

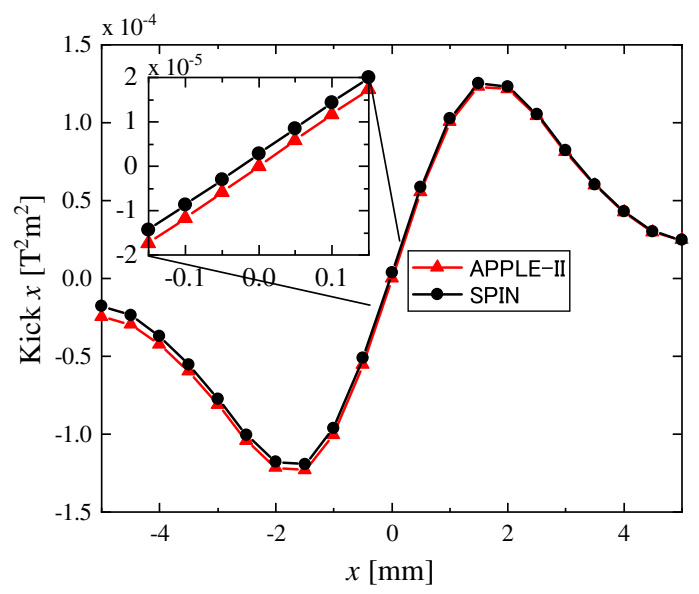

FIG. 8. Comparison between the APPLE and SPIN undulators in terms of horizontal kick map calculated at the gap of $4 \mathrm{~mm}$.

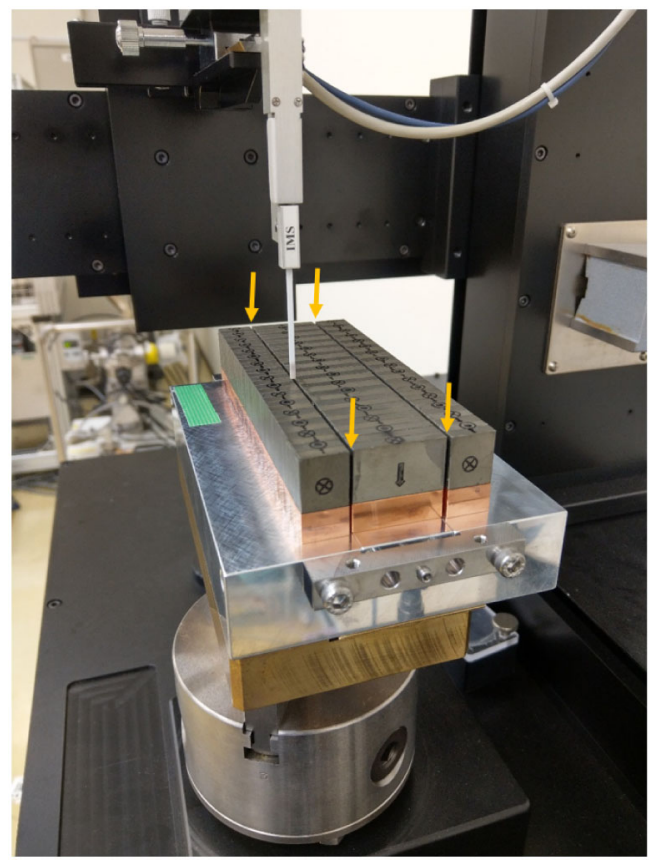

FIG. 9. Photograph of one of the two prototype magnetic arrays.

Figure 9 shows a photograph of one of the two prototype arrays; the magnetic field distribution was measured by moving a three-dimensional Hall sensor $2 \mathrm{~mm}$ above the surface along the RCP and LCP axes for each magnet array. The results were then summed up to evaluate the total magnetic field, which is expected when the two arrays are assembled to work as an undulator with the gap of $4 \mathrm{~mm}$.

The measurement results along the RCP and LCP axes are shown in Figs. 10(a) and 10(b). Figures 10(c) and 10(d) are the same as Figs. 10(a) and 10(b), but are shown as parametric plots, where we find that the magnetic field vector rotates clockwise/counterclockwise along the $\mathrm{RCP} / \mathrm{LCP}$ axis as expected.

Finally, we report the experimental optimization of the additional phase $\Delta \theta$ to minimize the magnetic force $\left(F_{y}\right)$. For this purpose, the magnetic arrays were assembled together to face with each other with the gap of $4 \mathrm{~mm}$. The magnetic force acting between the two magnetic arrays was measured by a load cell on which one of the two arrays was mounted. The measurement was repeated three times for different values of $\Delta \theta$, the result of which is summarized in Fig. 11. In this example, $F_{y}$ is expected to be minimized around $\Delta \theta=7^{\circ}$. This result is roughly consistent with the numerical result by RADIA as shown in the same figure; the small discrepancy may be attributable to a small misalignment of the side magnets in the longitudinal direction. Even so, the magnetic force $F_{y}$ can be reduced down to a level corresponding to the weight of the magnetic array (a few kilograms), which is practically sufficient. 

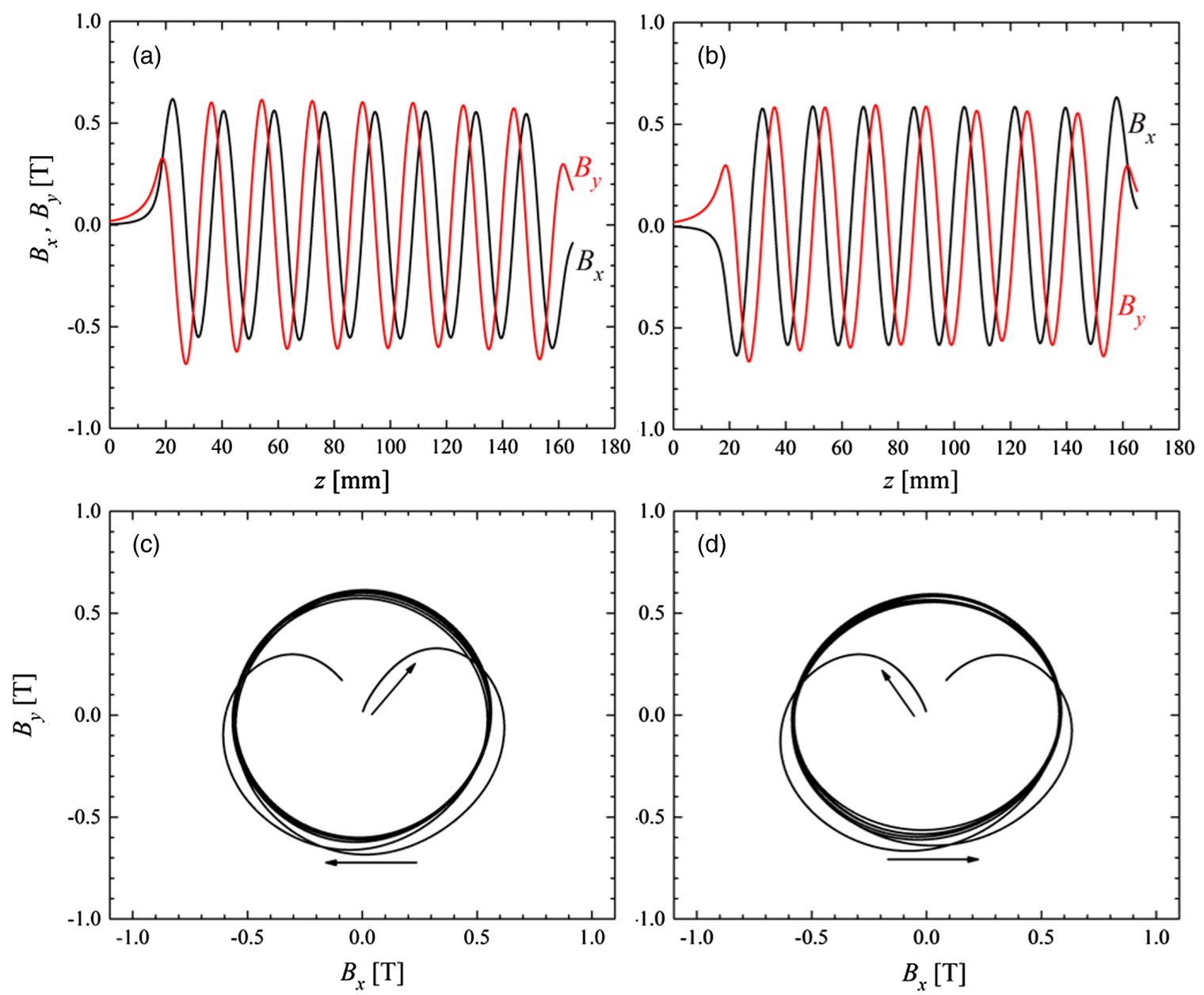

FIG. 10. Results of the field measurement: field distributions along the (a) RCP and (b) LCP axes, and their parametric plots (c) and (d), respectively.

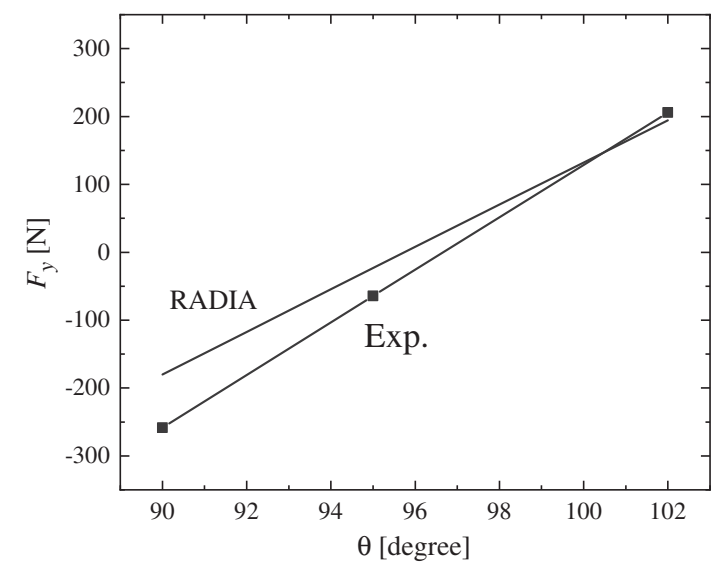

FIG. 11. Results of the force measurement, in comparison with the numerical ones.

\section{SUMMARY}

We proposed a new undulator configuration called SPIN to enable the helicity switching of circularly polarized radiation, and demonstrated its performance numerically and experimentally. The SPIN configuration is well compatible with IVUs because the phasing operation is not necessary and the magnetic force can be much weaker than conventional undulators.

Before closing, we mention another possibility of the SPIN undulator, although not discussed in detail in this paper; horizontally polarized radiation can be also generated by injecting the electron beam into the central axis, where only the vertical field survives and the horizontal field vanishes. This is obvious by recalling that the central array corresponds to a normal Halbach undulator with the relative phase of $90^{\circ}$ between the top and bottom arrays. Although the $\mathrm{K}$ value is $1 / \sqrt{2}$ times lower than the normal undulator with the same configuration, and vertically polarized radiation cannot be generated, it can be an attractive option of the SPIN undulator.

[1] K. J. Kim, A synchrotron radiation source with arbitrarily adjustable elliptical polarization, Nucl. Instrum. Methods Phys. Res. 219, 425 (1984). 
[2] S. Yamamoto and H. Kitamura, Generation of quasicircularly polarized undulator radiation with higher harmonics, Jpn. J. Appl. Phys. 26, L1613 (1987).

[3] S. Sasaki, K. Kakuno, T. Takada, T. Shimada, K.-i. Yanagida, and Y. Miyahara, Design of a new type of planar undulator for generating variably polarized radiation, Nucl. Instrum. Methods Phys. Res., Sect. A 331, 763 (1993).

[4] T. Tanaka and H. Kitamura, Figure-8 undulator as an insertion device with linear polarization and low on-axis power density, Nucl. Instrum. Methods Phys. Res., Sect. A 364, 368 (1995).

[5] K. Hirano, T. Ishikawa, and S. Kikuta, Development and application of x-ray phase retarders (invited), Rev. Sci. Instrum. 66, 1604 (1995).

[6] R. Walker, D. Bulfone, B. Diviacco, W. Jark, P. Michelini, L. Tosi, R. Visintini, G. Ingold, F. Schäfers, M. Scheer, G. Wüstefeld, M. Eriksson, and S. Werin, Design of an electromagnetic elliptical wiggler for ELETTRA, in Proceedings of PAC97, Vancouver, Canada, May 12-16, 1997 (JACoW, Geneva, Switzerland, 1997), pp. 3527-3529, https://doi.org/10.1109/PAC.1997.753264.

[7] T. Hara, T. Tanaka, T. Tanabe, X.-M. Maréchal, K. Kumagai, and H. Kitamura, SPring- 8 twin helical undulator, J. Synchrotron Radiat. 5, 426 (1998).

[8] T. Tanaka and H. Kitamura, Production of linear polarization by segmentation of helical undulator, Nucl. Instrum. Methods Phys. Res., Sect. A 490, 583 (2002).

[9] O. Marcouille, O. Chubar, A. Dael, and M.-P. Level, New designs of polarized electromagnetic undulators at SOLEIL, AIP Conf. Proc. 705, 207 (2004).
[10] J. Bahrdt and S. Grimmer, In-vacuum APPLE II undulator with force compensation, AIP Conf. Proc. 2054, 030031 (2019).

[11] O. Chubar, P. Elleaume, and J. Chavanne, A threedimensional magnetostatics computer code for insertion devices, J. Synchrotron Radiat. 5, 481 (1998).

[12] T. Hara, T. Tanaka, T. Tanabe, X.-M. Maréchal, S. Okada, and H. Kitamura, In-vacuum undulators of SPring-8, J. Synchrotron Radiat. 5, 403 (1998).

[13] T. Tanaka, X.-M. Maréchal, T. Hara, T. Tanabe, and H. Kitamura, Construction of a vertical undulator at SPring-8, J. Synchrotron Radiat. 5, 414 (1998).

[14] T. Hara, T. Tanaka, T. Seike, T. Bizen, X. Marchal, T. Kohda, K. Inoue, T. Oka, T. Suzuki, N. Yagi, and H. Kitamura, In-vacuum X-ray helical undulator for high flux beamline at SPring-8, Nucl. Instrum. Methods Phys. Res., Sect. A 467-468, 165 (2001), 7th Int.Conf. on Synchrotron Radiation Instrumentation.

[15] T. Tanaka, X.-M. Maréchal, T. Hara, T. Tanabe, and H. Kitamura, In-vacuum figure-8 undulator for hard X-rays with both horizontal and vertical polarization, J. Synchrotron Radiat. 5, 412 (1998).

[16] R. Kinjo, A. Kagamihata, T. Seike, H. Kishimoto, H. Ohashi, S. Yamamoto, and T. Tanaka, Lightweightcompact variable-gap undulator with force cancellation system based on multipole monolithic magnets, Rev. Sci. Instrum. 88, 073302 (2017).

[17] S. Takano, M. Masaki, K. Tamura, A. Mochihashi, T. Nakamura, S. Suzuki, M. Oishi, M. Shoji, Y. Taniuchi, Y. Okayasu, S. Okajima, and H. Ohkuma, Overview of the SPring-8 Diagnostics Beamlines, AIP Conf. Proc. 1234, 399 (2010). 